\title{
BIOÉTICA
}

CONTEXTO DA ODONTOLO HOSPITALAR NO BR 


\title{
BIOÉTICA NO \\ CONTEXTO DA ODONTOLOGIA HOSPITALAR NO BRASIL
}

\author{
ANDREA CRISTINA MARASSI LUCAS \\ UNIVERSIDADE FEDERAL DO PARÁ
}

KELLY LENE LOPES CALDERARO E U C LI DE S

UNIVERSIDADE FEDERAL DO PARÁ

L I L I A N E S L VA DO NASCIMEN T O

UNIVERSIDADE FEDERAL DO PARÁ

FLÁVIA SIROTHEAU CORREA PONTES

UNIVERSIDADE FEDERAL DO PARÁ

A M A N A MENEZES MEDEIROS

UNIVERSIDADE FEDERAL DO PARÁ

PETTRA B LA N C O L I RA

UNIVERSIDADE FEDERAL DO PARÁ 


\section{BIOÉTICA NO CONTEXTO DA ODONTOLOGIA HOSPITALAR NO BRASIL}

\section{Resumo}

O conceito de multidisciplinaridade é uma realidade crescente no cotidiano dos hospitais no Brasil. Neste sentido, vem ampliando na comunidade científica, estudos que evidenciam a relação direta entre a saúde bucal e a saúde geral do indivíduo. Desta forma, surge para o cirurgião-dentista o desafio de integração às equipes na prevenção e reabilitação dos pacientes internados nas unidades de terapia intensiva. As contribuições deste profissional são de suma importância para a melhoria da qualidade de vida e redução do tempo de internação dos pacientes, uma vez que muitas complicações podem ser minimizadas a partir da atuação da Odontologia no âmbito hospitalar. Neste contexto, há de se considerar a necessidade da Bioética, como ferramenta norteadora da assistência odontológica aos pacientes com necessidades especiais. Logo, o objetivo deste trabalho é realizar uma reflexão sobre a aplicação dos princípios básicos da Bioética na conduta do profissional de Odontologia hospitalar no Brasil.

Palavras-chave: Bioética, equipe hospitalar de odontologia, ética odontológica, assistência à saúde. 


\title{
BIOETHICS IN THE CONTEXT OF HOSPITAL DENTISTRY IN BRAZIL
}

\begin{abstract}
The concept of multidisciplinarity is a growing reality in the daily life of hospitals in Brazil. In this sense, studies that show the direct relationship between oral health and the general health of the individual have been increasing in the scientific community. Thus, the challenge of integrating teams in the prevention and rehabilitation of patients admitted to intensive care units arises to the surgeon-dentist. The contributions of this professional are of paramount importance for the improvement of the quality of life and reduction of the hospitalization time of the patients, since many complications can be minimized from the performance of the Dentistry in the hospital scope. In this context, the need for Bioethics must be considered as a guiding tool for dental care for patients with special needs. Therefore, the objective of this paper is to carry out a reflection on the application of the basic principles of Bioethics in the conduct of hospital Dentistry professionals in Brazil.
\end{abstract}

Keywords: Bioethics, hospital dentistry team, dental ethics, health care. 


\section{LA BIOÉTICA EN EL CONTEXTO DE LA ODONTOLOGÍA HOS- PITALARIA EN BRASIL}

\section{Resumen}

El concepto de multidisciplinariedad es una realidad creciente y cotidiana en los hospitales de Brasil. En este sentido, los estudios que evidencian la relación directa entre la salud bucal y la salud general del individuo han aumentado en la comunidad científica. De esta forma, surge para el cirujano-dentista el desafío de vincularse a los equipos de prevención y rehabilitación de los pacientes internados en las unidades de terapia intensiva. Las contribuciones de este profesional son de suma importancia para la mejoría de la calidad de vida y la reducción del tiempo de internación de los pacientes, ya que muchas complicaciones se pueden minimizar a partir de la actuación de la Odontología en el ámbito de los hospitales. En este contexto, debe considerarse la necesidad de la Bioética como herramienta que nortea la asistencia odontológica a los pacientes con necesidades especiales. Por tanto, el objetivo de este trabajo es realizar una reflexión sobre la aplicación de los principios básicos de la Bioética en la conducta del profesional en Odontología hospitalaria en Brasil.

Palabras Clave: Bioética, equipo hospitalario de odontología, ética odontológica, asistencia a la Salud.

Andrea Cristina Marassi Lucas

andreamarassi@yahoo.com.br

Kelly Lene Lopes Calderaro Euclides

kellycalderaro@hotmail.com

Liliane Silva do Nascimento

lilianenascimento2001@gmal.com

Flávia Sirotheau Correa Pontes

flaviapontes@ufpa.br

Amanda Menezes Medeiros

amanda mm12@hotmail.com

Pettra Blanco Lira

pettra_lira@yahoo.com.br 


\section{INTRODUÇÃO}

A integralidade é um dos princípios doutrinários da política de saúde do Estado brasileiro no Sistema Único de Saúde (SUS), o qual define o cuidado multiprofissional em todos os níveis assistenciais (Ministério da Saúde 2008: 92). Nessa perspectiva, a Odontologia se insere nos níveis de atenção básica, média e alta complexidade, sendo a resolução 162/2015 do Conselho Federal Odontologia (CFO) responsável por reconhecer o exercício da Odontologia Hospitalar $(\mathrm{OH})$ pelo cirurgião-dentista (CFO 2015).

As unidades de terapia intensiva (UTI) são voltadas ao cuidado do paciente em estado crítico, em todos os níveis de complexidade, exigindo assistência e observação contínua dos profissionais de saúde. O paciente de UTI encontra-se em situação vulnerável que abrange dificuldades familiares e psicossociais, que podem agravar o quadro clínico, logo, cuidados de caráter multidisciplinar se fazem necessários (CFO 2015; ABO 2016).

Entende-se como OH, o conjunto de ações preventivas, diagnósticas, terapêuticas, paliativas, de baixa, média ou alta complexidade, executadas em ambiente hospitalar, cujo objetivo é melhorar a saúde geral e a qualidade de vida do paciente internado. A abordagem integral do paciente é fundamental e depende da integração dos membros da equipe multidisciplinar que o assistem (CFO 2015; ABO 2016; Secretaria de Saúde 2012).

A cavidade bucal apresenta características que a colocam como um in- cubador microbiano ideal, por sofrer colonização contínua devido a grande variedade e concentração de populações microbianas. Várias espécies de microrganismos colonizam um ecossistema denominado biofilme, que apresenta alto potencial patogênico e possui proteção contra antimicrobianos (Jardim et al. 2013).

Neste sentido, a condição de higiene bucal, deficiente dos pacientes internados, pode desencadear diversos agravos bucais relacionados ao acúmulo de biofilme, como a cárie dentária, a xerostomia, a periodontite e a gengivite, os quais podem propiciar maior risco de complicações locais e sistêmicas (Pinheiro 2014). Entre as complicações, as mais prevalentes são a pneumonia bacteriana, a doença pulmonar obstrutiva crônica, as doenças cardiovasculares, a artrite reumatoide e os partos prematuros (Pinheiro 2014; Morais 2006).

A literatura aponta o aumento do número de pacientes que necessitam de tratamento odontológico, clínico ou cirúrgico, em ambiente hospitalar, nos últimos anos. Neste sentido, a $\mathrm{OH}$ vem ganhando destaque, superando barreiras advindas da cultura hospitalar, estabelecida entre a população envolvida, com o serviço. Fato este, relacionado com a carência na realização da higiene oral dos pacientes internados, tanto pela equipe de enfermagem, quanto pelos próprios acompanhantes (Jardim 2013; Morais 2006).

Logo, para que causas bucais não ocasionem consequências sistêmicas, é de suma importância a presença de um cirurgião-dentista no âmbito hospitalar, 
atuando no diagnóstico das alterações bucais, no tratamento e na adequação bucal do paciente crítico, de forma integrada à equipe multiprofissional (Jardim 2013; Pinheiro 2014; Padovani 2012).

Estudos comprovaram que o cuidado odontológico a pacientes hospitalizados contribui para a prevenção de agravos e melhora a condição sistêmica do paciente, reduzindo a incidência de infecções respiratórias do trato inferior, o uso de antimicrobianos sistêmicos, e consequentemente proporcionando a redução da mortalidade e do tempo de permanência no hospital. (Lindhe et al. 2005).

Observando a importância da atuação cirurgião-dentista no âmbito hospitalar para a melhoria da qualidade de vida do paciente internado, o Conselho Federal de Odontologia normatizou o exercício da Odontologia hospitalar por meio do código de ética odontológica (2012) no capítulo X (Da Odontologia Hospitalar), onde "Compete ao cirurgião-dentista internar e assistir paciente em hospitais..." e mais recentemente, através da Resolução CFO-162 (2015), onde são elencados os requisitos para a habilitação legal do cirurgião-dentista (CFO 2012; 2015).

Diante deste novo campo de atuação da Odontologia, se fazem necessárias algumas considerações, não só quanto à regulamentação, mas também na aplicação dos conceitos bioéticos no atendimento ao paciente hospitalizado. O sucesso de um tratamento clínico não pode estar relacionado apenas às características técnicas da equipe, deve-se considerar os princípios éticos que norteiam a equipe multidisciplinar, uma vez que a responsabilidade ética deve ser compartilhada (Freitas-Aznar et al. 2016).

Neste contexto, observa-se como desafio da equipe multiprofissional o cuidado integral dos pacientes internados, visando minimizar a dor e o sofrimento dos mesmos nas diversas dimensões, com diligências técnico-científica e humana, considerando as especificidades de cada caso individualizado (Freitas-Aznar 2016; Betinelli 2003).

Considerando a carência de pesquisas na área, nos propusemos a realizar uma descrição do histórico da ética odontológica hospitalar fomentando a reflexão sobre a aplicação dos princípios básicos da bioética (beneficência, não maleficência, autonomia e justiça) na conduta do profissional de Odontologia Hospitalar no Brasil.

\section{MATERIAL E MÉTODO}

Foi realizado estudo qualitativo-descritivo, por meio de levantamento bibliográfico, manual de livros e publicações a partir da base de dados PubMed, Scielo e BibliotecaVirtual em Saúde, no mês de Julho de 2018, utilizando os descritores "Bioética, equipe hospitalar de Odontologia, ética Odontológica e assistência à Saúde". Do total de 47 estudos, foram selecionados 25 artigos científicos, cujo critério de inclusão foram utilizados somente estudos que possuíam tema adequado ao objetivo proposto, dos quais 03 foram relatos de caso (12\%), 14 revisões de 
literatura (56\%), 01 estudo de corte transversal (4\%) e 07 manuais e resoluções de arcabouço legislativo (28\%), abordando a realidade nos hospitais, com a presença de cirurgiões-dentistas atuantes e a relevância da atuação dos mesmos nas unidades intensivas.

Foram selecionados também, artigos considerando e correlacionando as condições de saúde bucal e as condições sistêmicas dos pacientes internados, ressaltando a temática da Odontologia hospitalar e a reflexão dos princípios básicos da bioética que a regem. Não foram estabelecidos limites quanto ao ano, tipo de publicação e idioma. $\mathrm{O}$ total de 22 artigos foram excluídos por não serem condizentes aos critérios estabelecidos.

Foi realizada uma análise dos estudos selecionados, visando-se refletir sobre a importância do cirurgião-dentista no ambiente hospitalar, tanto no que tange à assistência técnica quanto da atuação dentro dos princípios da bioética, utilizando o histórico e os princípios como base norteadora do estudo, apoiada na legislação vigente.

\section{HISTÓRICO DA ÉTICA ODONTOLÓ- GICA HOSPITALAR}

Atualmente, com o aumento da expectativa de vida e sobrevida dos pacientes internados em hospitais no Brasil, principalmente a partir do desenvolvimento da medicina e da tecnologia nas três últimas décadas, a bioética tem sido amplamente discutida no que tange à questões de humanização e bem-estar nas relações interpessoais e de cuidado. Agravos e acidentes antes considerados letais, hoje são passíveis de tratamentos e intervenções que prolongam, de maneira significativa, a vida dos pacientes, suscitando a discussão e reflexão sobre a legitimidade e sobre o custo desse prolongamento da vida (Pontes et al. 2006; Espínola 2014; Godoi et al 2009). O código de ética odontológico afirma em seu capítulo X, artigo 26, que "compete ao cirurgião-dentista internar e assistir pacientes em hospitais públicos e privados, com ou sem caráter filantrópico, respeitadas as normas técnico administrativas das instituições". Frisando em seu artigo 27 que "as atividades odontológicas exercidas em hospital obedecerão às normatizações pertinentes". Descrevendo como infrações éticas: "I - fazer qualquer intervenção fora do âmbito legal da Odontologia; e, II - afastar-se de suas atividades profissionais, mesmo temporariamente, sem deixar outro cirurgião-dentista encarregado do atendimento e seus pacientes internados ou em estado grave" (Pinheiro 2017; ANVISA 2010).

Em 2004, foi instituída a ABRAOH, Associação Brasileira de Odontologia Hospitalar, que caracterizou a Odontologia hospitalar como ato odontológico exercido ao paciente internado que apresenta comprometimentos clínicos. A ABRAOH tem como objetivo: incentivar, regularizar e acompanhar a formação e a atuação dos odontólogos em ambiente hospitalar, auxiliando no reconhecimento deste campo de atuação (Pinheiro 2017; ABRAOH 2012).

Em 2005, o MS (Ministério da Saúde) concedeu ao cirurgião-dentista o 
direito a emitir a Autorização de Internação Hospitalar (AIH), através da portaria $\mathrm{N}^{\mathrm{o}} 1.011$, de 3 de outubro de 2004. Já no ano 2010, surge a resolução RDC nº que descreve os requisitos mínimos para o funcionamento de uma Unidade de tratamento intensivo (UTI), em seu artigo 18 instituindo a necessidade de haver diversos recursos assistenciais no leito, dentre eles, a assistência odontológica, que pode ser oferecida por meios próprios ou terceirizada. (Pinheiro 2017; ANVISA 2010; CFO 2003).

O artigo 21 da RDC n7, de 2010, expõe a importância do tratamento integral e interdisciplinar no nosocômio, onde se destaca a responsabilidade profissional da equipe no cuidado ao paciente, buscando o alívio do sofrimento e da dor, em todos os seus aspectos. Neste contexto a Bioética surge como instrumento norteador da atuação profissional, no sentido de difundir a necessidade de se pensar em perspectivas terapêuticas ampliadas, que possam ir além de cuidados medicamentosos e meras práticas clínicas de rotina, mas que possam considerar os aspectos pessoais, sociais, afetivos e culturais (Pinheiro 2017; ANVISA 2010).

Em 2013 foi criado o projeto de lei $n^{\circ} 34$, que se encontra em tramitação, e que torna obrigatória a prestação de assistência odontológica aos pacientes internados, portadores de doenças crônicas e os atendimentos em domicílio/ homecare. (Pinheiro 2017).

No ano de 2015, surge o reconhecimento da Odontologia Hospitalar como campo de atuação do cirurgião-dentis- ta a partir da resolução CFO-162/2015 do Conselho Federal de Odontologia, através da III Assembleia Nacional de Especialidades Odontológicas. Considerando o aumento crescente do número de profissionais habilitados para prestar assistência odontológica hospitalar, bem como o aumento da demanda reprimida, há de se refletir sobre a necessidade da aplicação da Bioética na orientação da conduta profissional dos mesmos (CFO 2015).

\section{PRINCÍPIOS BÁSICOS DA BIOÉTICA NA ODONTOLOGIA HOSPITALAR}

O cuidado com a dor e o sofrimento humano apresenta-se como grande desafio a ser trabalhado pela área da saúde, inclusive pelo cirurgião-dentista no ambiente hospitalar e aliar o conhecimento técnico-biológico aos princípios da Bioética, como a autonomia, a beneficência, a não maleficência e a justiça, é de suma importância na redução de riscos e melhoria da qualidade de vida do assistido (Pontes 2007; Silva et al 2013).

Refletindo sobre o princípio da beneficência na atuação da Odontologia hospitalar, o cirurgião-dentista deve se comprometer em avaliar tanto os riscos quanto os benefícios das ações realizadas, individual e/ou coletivamente, a fim de se reduzir a exposição dos pacientes a riscos e danos, bem como avaliar a auto-percepção de Saúde bucal e condições sócio-econômicas do paciente, antes de iniciar as orientações, prescrições e adoção de procedimentos pertinentes à sua atuação (Freitas-Aznar et al. 2016; Pontes 2007). 
Sobre a não maleficência, o profissional deve, além de evitar danos à integridade do paciente, evitar a prática de atos embasados na culpa, em danos previsíveis, advindos de terapêuticas adotadas por negligência, imprudência ou imperícia, que podem acarretar em males ao paciente (Freitas-Aznar et al. 2016; Pontes 2007).

A negligência ou falta de cuidados necessários para a higiene oral do paciente acamado, inconsciente e/ou impossibilitado de realizá-la por si próprio, pode ferir o princípio da não maleficência, uma vez que agravos respiratórios como a Pneumonia associada à ventilação (PAV) podem se instalar após 48 horas de internação em UTI, devido ao acúmulo de agentes patogênicos respiratórios na cavidade oral (Freitas-Aznar et al. 2016; Pinheiro 2014; Morais 2006).

O princípio da autonomia pode ser definido como o direito de um indivíduo em exercer seu protagonismo no seu processo de saúde e doença. Logo, a autonomia está ligada à dignidade da pessoa humana, devendo-se resguardar a liberdade de decisão de cada indivíduo. Neste pressuposto, há de se garantir, no campo da Odontologia hospitalar, o respeito às escolhas do paciente internado e/ou familiares, quando pertinente, priorizando a autonomia do mesmo, em detrimento às convicções terapêuticas do profissional (Freitas-Aznar et al. 2016; Baère 2017).

O consentimento livre e esclarecido tem sido uma ferramenta bastante utilizada pelas equipes odontológicas nos hospitais brasileiros visando o respeito à autonomia dos pacientes, além da garantia do exercício da beneficência para com o paciente internado.

Em uma pesquisa realizada com pacientes de oito hospitais do Distrito Federal, os autores evidenciaram a utilização pelas equipes odontológicas de documento escrito e/ou acordo verbal entre profissionais e pacientes. Os autores concluíram que a utilização de forma consciente deste documento é capaz de assegurar a autonomia, além de resguardar a conduta profissional do cirurgião-dentista (Freitas-Aznar et al. 2016; Baère 2017).

A humanização da saúde dentro do ambiente hospitalar pode simplificar as ações, medidas adotadas e comportamentos que podem ser adotados na garantia da dignidade dos pacientes internados. A ética deve ser o elemento propulsor dos procedimentos e intervenções pessoais e profissionais, instrumentos importantes na construção do processo de humanização na assistência à saúde (Freitas-Aznar et al. 2016; Silva et al. 2013; Lima \& Souza 2010).

Neste contexto, todos os profissionais de saúde da equipe devem propiciar o acesso e a assistência humanizada aos pacientes, com o apoio do Serviço Social do hospital, que tem por objetivo fazer o acolhimento do indivíduo e da família, conforme a demanda apresentada, e, assim, proporcionar um atendimento individualizado e diferenciado, garantindo a orientação e o encaminhamento necessários. (Xavier e Lopes, 2016)

É de suma importância a interface Odontologia/Serviço Social, no que 
tange à avaliação das necessidades específicas do paciente internado em relação às condições e agravos bucais, muito relacionados à falta de acesso à serviços de saúde, ao saneamento básico, a métodos preventivos contra a cárie e a doença periodontal, a moradia adequada, além de questões de vulnerabilidade sócio-econômica, de negligência familiar, violência, entre outras que dependem de um olhar multiprofissional para o seu enfrentamento.

No princípio da justiça, a prioridade do profissional é avaliar as necessidades dos indivíduos, sendo resguardadas suas diferenças e singularidades, isto é, o exercício da imparcialidade. Logo, o profissional deve fomentar sua atuação na avaliação da relevância social e do sentido de sua destinação sócio humanitária. Neste sentido a conduta do cirurgião-dentista nas UTI(s) pode contribuir para a promoção da justiça no âmbito nosocomial, uma vez que o mesmo atua no diagnóstico de agravos bucais, alívio de sintomas e redução de danos, resultando na melhoria da qualidade de vida dos pacientes (Freitas-Aznar et al. 2016; Pinheiro 2017).

\section{CONCLUSÃO}

A Odontologia hospitalar, campo de atuação crescente nos hospitais do Brasil a partir da RDC no 7 de 2010, tem demonstrado relevância na redução da morbi-mobilidade hospitalar, principalmente no que tange às doenças respiratórias como a Pneumonia associada à Ventilação (PAV), bem como na redução da permanência do paciente internado e melhoria da qua- lidade de vida do paciente internado. Neste sentido, a prática da assistência odontológica nos hospitais deve considerar o cuidado integral do paciente, de forma integrada e multidisciplinar, priorizando o atendimento humanizado e digno.

Embora os estudos comprovem a relevância da atuação multiprofissional nas UTI(s), o que se observa é que ainda existem muitas limitações no trabalho em equipe, principalmente na construção de planos terapêuticos e na área de pesquisa científica, uma vez que se observa que a maioria dos estudos existentes possuem autoria segmentada por categorias profissionais, ocorrendo pouca interação entre os diversos campos científicos.

É importante ressaltar a escassez de trabalhos científicos sobre o histórico da Bioética na Odontologia hospitalar e a aplicação dos princípios bioéticos na atuação dos cirurgiões-dentistas nos hospitais brasileiros, no entanto se faz necessário incentivar a reflexão sobre a importância dos pilares bioéticos da autonomia, justiça, beneficência e não maleficência na fundamentação da assistência odontológica no âmbito hospitalar.

\section{REFERÊNCIAS:}

Associação Brasileira de Odontologia Hospitalar (ABRAOH), Estatuto Social da Associação Brasileira de Odontologia Hospitalar. 2013. Disponível em: $<\underline{\text { ht- }}$ tps://abraoh.wordpress.com>. Acesso em: 09 julho. 2018.

Associação Brasileira de Odontologia Hospitalar. 2013. Disponível em: < $\underline{\text { http:// }}$ 
www.abraoh.org.br/ quem-somos/>. Acesso em 10 julho. 2018.

Agência Nacional de Vigilância Sanitária. Resolução-RDC $n^{\circ}$ 7. 2010. Disponível em: <URL: http://www.amib.org.br/pdf/ RDC-07-2010.pdf $>$. Acesso em 10 julho. 2018.

Amaral, S. M., Cortes A. Q e Pires F. R. 2009. Pneumonia nosocomial: importância do microambiente oral. J Bras Pneumol 35:1116-24.

Baère, T. D e Miranda A. F. 2017. A importância da prática interdisciplinar da equipe de saúde nos cuidados paliativos. Revista Portal de Divulgação 53:5-19.

BRASIL. Ministério da Saúde. Secretaria de Atenção à Saúde. Departamento de Atenção Básica, Saúde Bucal. 2008. Normas e Manuais Técnicos - Cadernos de Atenção Básica. Brasília.

Bettinelli, L. A., Waskievicz J e Erdmann A. L. 2003. Humanização no ambiente do cuidado hospitalar. O Mundo da Saúde 27:231-9.

Consalter, C. E., Rodrigues S. O. 1999. Papel da higiene bucal na diminuição do desenvolvimento de pneumonia em pacientes sob cuidados de UTI. Smina Londrina 20:13-20.

Conselho Federal de Odontologia. Código de Ética. Resolução CFO-42. 2003. Disponível em: $<$ www.cfo.org.br/download/ pdf/codigo etica.pdf $>$. Acesso em 11 julho. 2018.

Conselho Federal de Odontologia. Código de Ética Odontológica. Resolução CFO 118/2012. 2013. [Internet]. Disponível em: $<$ http://cfo.org.br/legislacao/codigos/>. Acesso em 10 julho. 2018.

Conselho Federal de Odontologia. 2015. Resolução 162. Diário Oficial da União. 1: 167.
Freitas-Aznar, A. R., Capelozza, A. L. A., Costa Aznar, F. D., Junior, L. A. V. S e Santos, P. S. S. 2016. A bioética no contexto da Odontologia Hospitalar: uma revisão crítica. Rev. bras. Odontol. 4:311-314.

Godoi, A. P. T., Francesco, A. R., Duarte, A., Kemp, A. P. T e Silva-Lovato, C. H. 2009. Odontologia Hospitalar no Brasil: Uma visão Geral. Rev Odontol UNESP 38:105-9.

Governo do Estado de São Paulo, Secretaria de Saúde de São Paulo. 2012. Manual de odontologia hospitalar. São Paulo.

Jardim, E. G., Setti, J. S., Cheade, M. F. M e Mendonça, J. C. G. 2013. Atenção Odontológica a pacientes hospitalizados: Revisão da Literatura e Proposta de Protocolo de Higiene Oral. Rev. Brasileira de Ciências da Saúde 35:31-36.

Lima, E. N. A e Souza, E. C. F. 2010. Percepção sobre ética e humanização na formação odontológica. Rev Gaúcha Odontol. 2:231-238.

Lindhe, J., Karring, T e Lang, N. P. 2005. Tratado de Periodontia Clínica e Implantologia Oral. pp 80-104. Rio de Janeiro: Guanabara Koogan.

Morais, T. M. N., Silva, A., Avi, A. L. R. O., Souza, P. H. R., Knobel, E e Camargo, L. F. A. 2006. A Importância da Atuação Odontológica em Paciente internados em Unidade de Terapia Intensiva. Revista Brasileira de Terapia Intensiva 4:412-417.

Oliveira, L. C. B. S., Carneiro, P. P. M., Fischer, R. G e Tinoco EMB. 2007. A Presença de Patógenos Respiratórios no Biofilme Bucal de Pacientes com Pneumonia Nosocomial 19: 428-433.

Padovani, M. C. R. L., Souza, S. A. B., Santanna, G. R e Guaré, R. 2012. O Protocolo de cuidados bucais na Unidade de Tratamento Intensivo (UTI). Neonatal. Revista Bras.de Pesquisa em Saúde 1:71-80. 
Pinheiro, T. B. 2017. Odontologia Hospitalar: A importância do cirurgião-dentista nas unidades de terapia intensiva e sua atuação no atendimento a pacientes portadores de necessidades especiais. 1-27. Trabalho de conclusão de curso apresentado ao Centro Universitário São Lucas, Porto Velho, Brasil.

Pinheiro, T. S e Almeida, T. F. 2014. A Saúde Bucal em Pacientes de UTI. Revista Babiana de Odontologia 2:94-103.

Pontes, A. C., Espíndula, J. A., do Valle, E. R. M e Santos, M. 2007. Bioética e profissionais de saúde: algumas reflexões. Revista Bioethikos Centro Universitário São Camilo 1:68-75.

Silva, A. M., Sá, M. C e Miranda, L. 2013. Concepções de sujeito e autonomia na humanização em saúde: uma revisão bibliográfica das experiências na assistência hospitalar. Saúde Soc. São Paulo 3:840-852.

Xavier, P. R. H e Lopes, S. M. B. 2016. As práticas do Assistente Social e sua articulação com a Equipe de Saúde de um Hospital de Curitiba. Serv. Soc. Rev., Londrina. 2:132 - 150 . 